\title{
Chapter 7 Dynamic Modeling, Simulation and Velocity Control of Rocker-Bogie Rover for Space Exploration
}

\author{
Pushpendra Kumar \\ Indian Institute of Technology Roorkee, India \\ Pushparaj Mani Pathak \\ Indian Institute of Technology Roorkee, India
}

\begin{abstract}
Wheeled mobile rovers are being used in various missions for planetary surface exploration. In this paper a six-wheeled rover with rocker-bogie structure has been analyzed for planar case. The detailed kinematic model of the rover was built and the dynamic model was derived based on bond graph. The simulation studies were performed for obstacle climbing capability of the rover. It was observed from the study that rover can pass through plane surface, inclined surface, and inclined ditch without any control on the actuators of the rover. However, it fails to cross a vertical ditch so a velocity controller was designed. It consists of a proportional integral (PI) controller and reduced model of the rover. It is found from simulation and animation studies that with the proposed velocity controller the rover is able to cross the vertical ditch.
\end{abstract}

\section{INTRODUCTION}

Many planetary exploration rovers have been developed since 1960s for planetary exploration. Based on the features of the mobile mechanism in robots, planetary exploration rovers have various types such as wheeled, legged and tracked.
Wheeled mobile mechanisms have excellent features, such as high speed on a relatively flat terrain and easy control; so many researchers have designed their exploration rovers with wheeled structure. Wheeled structure planetary rovers have four-wheel, six-wheel and eight-wheel, etc. Among these rovers, the six-wheeled mobile rover

DOI: 10.4018/978-1-4666-3634-7.ch007

Copyright $@$ ( 2013, IGI Global. Copying or distributing in print or electronic forms without written permission of IGI Global is prohibited. 
Figure 1. Schematic diagram of rocker bogie rover

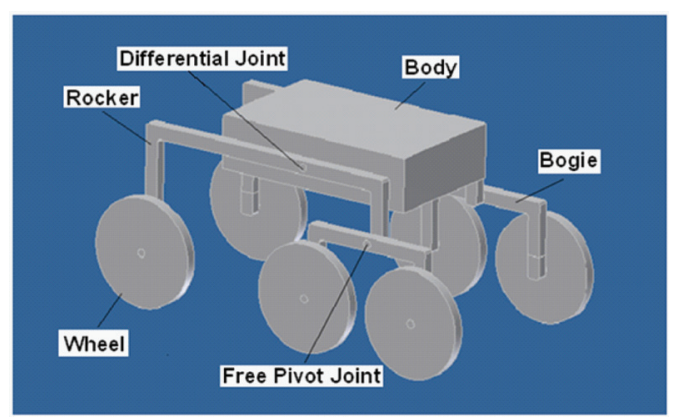

with rocker bogie mechanism has superior adaptability and obstacle climbing capability. These rovers have been used in Mars Rovers (Lindemann, Bickler, Harrington, Ortiz, \& Voorhees, 2006; Lindemann, 2005).

The research in exploration rovers has been devoted to analyze the performance of exploration rovers based on different types of suspension systems. Bai-chao, Rong-ben, Lu, Li-sheng, and Lie (2007) proposed a new type of suspension system formed by a positive and a negative quadrilateral levers mechanism. Thueer, Siegwart, and Backes, Planetary Vehicle Suspension Options (2008) studied the locomotion performance of different suspension types in order to find the rover that matches best for any mission requirement. Their performance was evaluated on hard and loose soil. Thueer, Krebs, Siegwart, and Lamon (2007) presented the performance optimization tool based on a static approach to compare and optimize the rover chassis in quick and efficient way. Thueer and Siegwart (2007) analyzed different rovers based on kinematic model and the optimal velocities of all wheels were used for characterization of the suspension of different rovers. Hacot, Dubowsky, and Bidaud (1998) presented the mechanics of rover and method for solving the inverse kinematics of the rocker-bogie rover. The quasi-static force analysis has been described for the rover. $\mathrm{Li}, \mathrm{Gao}$, and Deng (2008) built the mobility performance indexes based on work conditions and established an optimized mathematical model of suspension parameters of rocker-bogie rover. Mann and Shiller (2005) described a measure of stability of rockerbogie rover for a range of acceptable velocities and accelerations that satisfy a set of dynamic constraints. Yongming, Xiaoliu, and Wencheng (2009) described the quasi-static mathematical model of rocker-bogie rover. Obstacle climbing capabilities of the rover were analyzed. Most of the rover models reported in literature is based on kinematic relations. Rover dynamic relations have not been derived.

In this paper the kinematic model of the rocker-bogie rover is developed. This kinematic model is used in deriving the dynamic model using bond graph technique (Mukherjee, Karmakar, \& Samantaray, 2006; Karnopp, Margolis, \& Rosenberg, 2000). The dynamic model includes the model of the motor and ground reaction. The

Figure 2. Planar model of rocker bogie rover

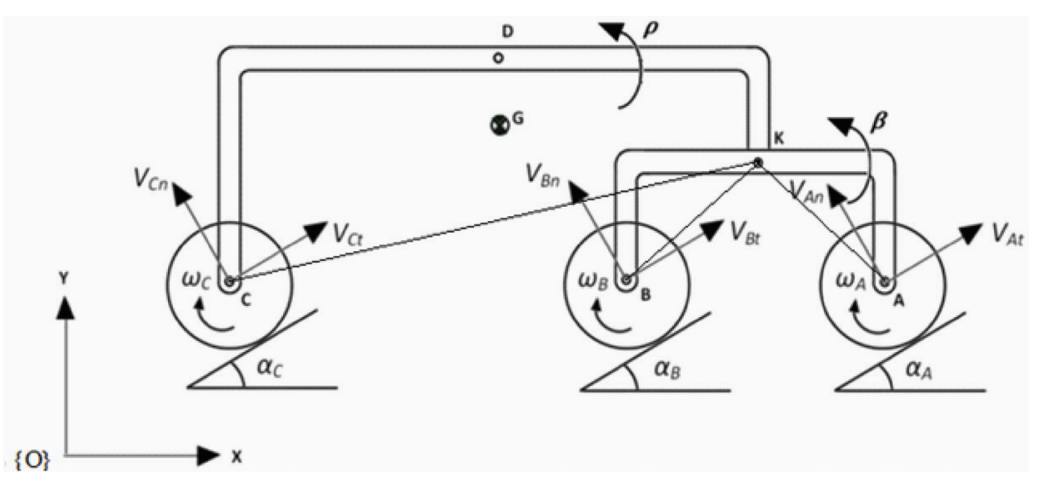


13 more pages are available in the full version of this document, which may be purchased using the "Add to Cart" button on the publisher's webpage: www.igi-global.com/chapter/dynamic-modeling-simulation-velocitycontrol/76442

\section{Related Content}

\section{Advanced SLAM Techniques}

(2013). Simultaneous Localization and Mapping for Mobile Robots: Introduction and Methods (pp. 336389).

www.irma-international.org/chapter/advanced-slam-techniques/70688/

Re-Broadcast AODV(reAODV) Based Routing Protocol Modification Over AODV for VANET In City Scenario

Soumen Saha, Utpal Roy and D.D. Sinha (2018). International Journal of Synthetic Emotions (pp. 51-65). www.irma-international.org/article/re-broadcast-aodvreaodv-based-routing-protocol-modificationover-aodv-for-vanet-in-city-scenario/209426/

Portable Haptic Arm Exoskeleton

Pierre Letier and André Preumont (2012). Prototyping of Robotic Systems: Applications of Design and Implementation (pp. 122-145).

www.irma-international.org/chapter/portable-haptic-arm-exoskeleton/63533/

In Defense of Dominance: PAD Usage in Computational Representations of Affect Joost Broekens (2012). International Journal of Synthetic Emotions (pp. 33-42).

www.irma-international.org/article/defense-dominance-pad-usage-computational/66088/

Novel Swimming Mechanism for a Robotic Fish

Sayyed Farideddin Masoomi, XiaoQi Chen, Stefanie Gutschmidt and Mathieu Sellier (2013). Engineering Creative Design in Robotics and Mechatronics (pp. 41-58).

www.irma-international.org/chapter/novel-swimming-mechanism-robotic-fish/78098/ 